PACS: 98.80.-k, 98.80.Es

\title{
COSMOGRAPHY OF THE DYNAMICAL COSMOLOGICAL «CONSTANT»
}

\author{
(D) Oryna Ivashtenko \\ V. N. Karazin Kharkiv National University, Kharkiv, Ukraine \\ Svobody Sq. 4, Kharkiv, Ukraine, 61022 \\ E-mail: arinaivashtenko@gmail.com \\ Received 31 October 2018, revised 12 November 2018
}

The paper considers a cosmographical approach to analyze cosmological models. Cosmography is a method to describe the kinematics of the cosmological expansion based only on the cosmological principle. We consider a method of treating free parameters of a cosmological model in terms of the directly observable cosmographic values related to the time-derivatives of the Hubble parameter (deceleration, jerk, snap). The method is applied to analyze two cosmological models involving the timedependence of the cosmological constant in the form $\Lambda(t) \rightarrow \Lambda(H)$ when this approach is especially efficient. Both models interpret the dark energy in the form of the cosmological constant $\Lambda$ as energy of physical vacuum, which is currently the most supported treatment. The first one means $\Lambda$ being proportional to the Hubble parameter, and the second one involves a constant and a quadratic term. As a result, the free parameters of both models are expressed in terms of the currently observed values of the Hubble parameter, deceleration, and jerk. The obtained expressions for model parameters are exact, as the method does not involve any additional assumptions. Furthermore, it leads to deal with algebraic equations instead of differential ones. After this procedure, solutions of the evolution equations are obtained in the form of the time-dependence of the Hubble parameter. The obtained model parameters are substituted to the solutions, which are analyzed for a typical range of cosmographic scalars taken from recent observations. Finally, the proposed approach is used to eliminate free parameters from both models and to obtain constraints for the directly observable cosmographic values that can be tested to correspond to present observations data. For the considered cases, such constraints are received respectively for the jerk and the snap parameters with respect to the deceleration. The constraint for the linear model is compared with current observational value ranges for the deceleration and the jerk parameters.

KEYWORDS: cosmography, cosmographic parameters, cosmological constant

\section{КОСМОГРАФІЯ ДИНАМІЧНОЇ КОСМОЛОГІЧНОЇ КОНСТАНТИ О. Іваштенко}

Харківський національний університет ім. В. Н. Каразіна, Харків, Украӥна м. Свободи 4, м. Харків, Україна, 61022

У цій роботі розглянуто космографічний підхід до аналізу космологічних моделей. Космографія - це спосіб опису кінематики космологічного розширення, що базується лише на космологічному принципі. Ми розглядаємо метод, який полягає у вираженні вільних параметрів космологічної моделі в термінах космографічних величин, пов'язаних 3 похідними за часом від параметра Хабла (q, j, s), що доступні для безпосередніх спостережень. Спосіб застосовано для аналізу двох космологічних моделей, що включають залежність космологічної сталої від часу у формі $\Lambda(t) \rightarrow \Lambda(H)$, коли такий підхід $\epsilon$ особливо ефективним. Обидві моделі трактують темну енергію у формі космологічної константи $\Lambda$ як енергію фізичного вакууму, що наразі являє собою найбільш розповсюджений підхід. Перша модель включає в себе лінійну залежність $\Lambda$ від параметра Хабла, а друга містить константу та квадратичний за $H$ доданок. В результаті вільні параметри обох моделей виражено через поточні значення космографічних величин, які можуть бути отримані зі спостережень. Отримані вирази для модельних параметрів є точними, оскільки метод не вимагає ніяких додаткових наближень. Окрім того, він дозволяє працювати з алгебраїчними рівняннями замість диференційних. Після цього рішення еволюційних рівнянь знайдено у формі залежності від часу параметра Хабла. Отримані параметри моделей підставлено до рішень, які проаналізовано для характерного діапазону космографічних величин, взятого з нещодавніх спостережень. Врешті решт, запропонований підхід дозволяє виключити вільні параметри з обох моделей та отримати зв'язки для космографічних величин, що можуть бути отримані зі спостережень. Вони надалі можуть бути протестовані на відповідність сучасним експериментальним даним. Для розглянутих випадків такі співвідношення отримано відповідно для параметрів j та s відносно параметру q. Обмеження для лінійної моделі порівняно з поточними діапазонами значень для цих параметрів зі спостережень.

КЛЮЧОВІ СЛОВА: космографія, космографічні параметри, космологічна стала

\section{КОСМОГРАФИЯ ДИНАМИЧЕСКОЙ КОСМОЛОГИЧЕСКОЙ ПОСТОЯННОЙ А. Иваштенко \\ Харьковский национальный университет имени В. Н. Каразина, Харьков, Украина пл. Свободы 4, г. Харьков, Украина, 61022}

В работе рассмотрен космографический подход к анализу космологических моделей. Космография - это способ описания кинематики космологического расширения, основанный только на космологическом принципе. Мы рассматриваем метод, заключающийся в выражении свободных параметров космологических моделей в терминах непосредственно наблюдаемых космографических величин, связанных с производными по времени от параметра Хаббла (параметры q, j, s). Метод применён для анализа двух космологических моделей с космологической константой, зависящей от времени в форме $\Lambda(t) \rightarrow$ $\Lambda(H)$, когда этот подход особенно эффективен. Обе модели интерпретируют темную энергию в форме космологической 
постоянной $\Lambda$ как энергию физического вакуума, что в настоящее время является самым распространённым подходом. Первая модель рассматривает линейную зависимость $\Lambda$ от параметра Хаббла $H$, а вторая включает константу и квадратичное по $H$ слагаемое. В результате свободные параметры обеих моделей выражены в терминах текущих значений космографических переменных. Полученные выражения для модельных параметров являются точными, так как метод не требует никаких дополнительных приближений. Кроме того, он позволяет работать с алгебраическими уравнениями вместо дифференциальных. После этого решения эволюционных уравнений найдены в форме зависимости от времени параметра Хаббла. Полученные параметры моделей подставлены в решения, которые проанализированы для характерных диапазонов значений космографических параметров, взятых из недавних наблюдений. Наконец, предложенный подход позволяет исключить свободные параметры из обеих моделей и получить связи на наблюдаемые значения космографических величин, которые могут быть протестированы на соответствие с современными наблюдательными данными. Для рассмотренных случаев такие связи получены соответственно для параметров j и s по отношению к параметру q. Соотношение для линейной модели сравнивается с текущим наблюдаемым диапазоном значений этих величин.

КЛЮЧЕВЫЕ СЛОВА: космография, космографические параметры, космологическая постоянная

The Standard Cosmological Model (SCM) [1] describing a homogeneous and isotropic spatially flat accelerating expanding universe is in a good agreement with a huge number of the observational data [2]. The accelerated expansion in this model is generated by a substance with the negative pressure, named the cosmological constant (CC). SCM succeeded to resolve the main problems of the previous cosmological model (the Big Bang model) [3].

However, the great success of the SCM for which it even got a name Cosmic Concordance should not be misleading. The Cosmological model whereby our world consists $95 \%$ of entities of unknown nature (the dark energy $\sim 70 \%$, and the dark matter $\sim 25 \%$ ) can be only an intermediate stage on the way to understanding the structure of the Universe.

The lack of the information on the nature of main components of the Universe energy budget significantly increases the number of possible ways to describe the cosmological evolution. The transfer from the cosmological constant to dynamical forms of the dark energy is one of the most popular ways to generalize the SCM. The simplest example of this type models is given by models with decaying vacuum. These models assume the energy exchange in the dark sector.

The relation between the components will lead to the modification of evolution of the Universe. Particularly, the dark energy density (in the form of cosmological «constant») will not remain constant. Such a modification vastly expands the possibilities of the model and allows resolving a number of conceptual problems of the SCM, in particular the problem of cosmic coincidence [4]. Unfortunately, as it often happens when introducing a new model, a serious universal problem occurs in determining parameters of the model.

This problem can be resolved by reconstruction of parameters of cosmological models based on using their kinematic characteristics [5,6]. The traditional kinematics of the Universe works in accordance with the cosmological principle stating that on scales exceeding hundreds of mega parsecs the Universe is homogeneous and isotropic. A method to describe the evolution of the Universe named cosmography is based only on the cosmological principle and presents actually a kinematics of the cosmological expansion. The efficiency of the cosmography is that it allows expressing parameters of any model satisfying the cosmological principle, in terms of a limited number of cosmographic parameters (first few Taylor series expansion coefficients of the scale factor).

In the present work, we use cosmography to find parameters of two models of the dynamical cosmological constant.

\section{MODELS}

At the present time, the interpretation of the dark energy in the form of the cosmological constant $\Lambda$ as energy of physical vacuum is the most supported among other alternatives. It automatically leads to the equation of state of this substance $p_{\Lambda}=-\rho_{\Lambda} \quad\left(p_{\Lambda}\right.$ is the pressure, and $\rho_{\Lambda}$ is the energy density), ensuring the accelerating expansion of the Universe. The hypothesis allowing resolving a number of current cosmological problems involves moving to a timedependent $\mathrm{CC}, \Lambda \rightarrow \Lambda(t)$. In virtue of the energy conservation law, the vacuum decay should be accompanied by changing the dark matter energy density $\rho_{m}$. Dynamics of the two-component system can be described by the system of equations comprising the first Friedmann equation and the conservation equation (we use a system of units where the reduced Planck mass $\left.M_{P l}=(8 \pi G)^{-1 / 2}=c=1\right)$

$$
\begin{aligned}
& \rho_{m}+\rho_{\Lambda}=3 H^{2}, \\
& \dot{\rho}_{m}+3 H \rho_{m}=-\dot{\rho}_{\Lambda},
\end{aligned}
$$

where $H$ is the Hubble parameter, and we took into account that for the cold dark matter the pressure $p_{m}=0$. In the right part of the conservation equation there is a new term $\dot{\rho}_{\Lambda}$ playing the role of the source generated by the decaying CC. From the system (1) one can obtain the equation for the Hubble parameter

$$
2 \dot{H}+3 H^{2}-\rho_{\Lambda}=0 .
$$


At the phenomenological level to solve the equation (2) a model of the CC decay is needed. Below, we will consider a simple, but fully analyzable model [7], where

$$
\rho_{\Lambda}=\sigma H
$$

We will show that cosmography allows us to easily express a single parameter of the model $\sigma$ in terms of the cosmographic parameter, namely the deceleration.

The two-parametric model suggested in the work [8] also treats the $\mathrm{CC}$ as the decaying vacuum energy. More specifically, the ideology of this model goes back to the hypothesis about existence of an unstable false vacuum [9-11]. If $E_{0}^{(f a l s e)}$ and $E_{0}^{(t r u e)}$ are energies of the false and the true vacuums, the hypothesis involves a universal behavior of the substance initially having been in the false vacuum

$$
E_{0}^{(\text {false })}=E_{0}^{(\text {true })}+\frac{\alpha}{t^{2}} \pm \cdots \quad \text { for } t \gg T
$$

where $T$ is the typical time of the tunneling from the false vacuum to the true one. In terms of the time-depending CC the relation (4) can be rewritten as [8]

$$
\rho_{\Lambda}(t)=\Lambda(t)=\Lambda_{\text {bare }}+\frac{\alpha}{t^{2}}
$$

that emerges from the covariant theory of a scalar field and presents a leading term at the late time of evolution.

\section{METHOD}

Characteristics used to describe the evolution of the universe can be either kinematic or dynamical. The first ones presenting different combinations of time-derivatives of the scale factor are model-independent. While the second-ones to be found require, besides dynamical equations of GTR, models of the equations of state of the considered components. Like in the classical mechanics, cosmokinematics describes the observed evolution of the Universe without respect to its causes. The kinematics of cosmological expansion of a homogeneous and isotropic universe has been called cosmography [12].

In the early 60s, Alan Sandage [13] defined as the primary goal of the cosmologists a search for two parameters, namely, the Hubble parameter and the deceleration parameter. However, an expansion with a constant acceleration is not the only possible realization of the kinematics of a nonstationary universe. As the universe evolves, the relative content of the components that fill it is changing, and as consequence, the dynamics of expansion changes, hence the changes in acceleration. Thus, for a more complete description of the kinematics of cosmological expansion, it is useful to consider an extended set of parameters (Hubble $H$, deceleration $d$, jerk $j$, and snap $s$ ) by including a higher-order temporal derivatives of the scale factor [14]

$$
\begin{gathered}
H(t) \equiv \frac{1}{a} \frac{d a}{d t} \\
q(t) \equiv-\frac{1}{a} \frac{d^{2} a}{d t^{2}}\left[\frac{1}{a} \frac{d a}{d t}\right]^{-2} \\
j(t) \equiv \frac{1}{a} \frac{d^{3} a}{d t^{3}}\left[\frac{1}{a} \frac{d a}{d t}\right]^{-3} ; \\
s(t) \equiv \frac{1}{a} \frac{d^{4} a}{d t^{4}}\left[\frac{1}{a} \frac{d a}{d t}\right]^{-4}
\end{gathered}
$$

The inclusion of higher derivatives of the scale factor, on the one hand, reflects the continuous progress of observational cosmology, and, on the other hand, is dictated by the need to describe the increasingly complex effects used for obtaining precise information.

In 2008, Dunajski and Gibbons [15] proposed an original approach for testing cosmological models satisfying the cosmological principle. The implementation of this method involves the following sequence of steps:

1. The first Friedmann equation is transformed to the ODE for the scale factor. This transformation is achieved by using the conservation equation for each component included in the model.

2. The resulting equation is differentiated (with respect to cosmological time) as many times as the number of free parameters of the model. 
3. The time derivatives of the scale factor are expressed through the cosmographic parameters.

4. By solving the obtained system of linear algebraic equations, all the free parameters of the model are expressed through the cosmographic parameters.

The procedure under consideration can be made more universal and effective if the system of Friedmann equations for the Hubble parameter $H$ and its time derivative $\dot{H}$ is considered as a starting one [6]. By differentiating the equation the required number of times (determined by the number of free parameters of the model), we obtain a system of equations including higher derivatives of the Hubble parameter $H$. These derivatives are directly related to the cosmographic parameters by the relations

$$
\begin{aligned}
& \dot{H}=-H^{2}(1+q) ; \\
& \ddot{H}=H^{3}(j+3 q+2) ; \\
& \dddot{H}=H^{4}(s-4 j-3 q(q+4)-6) .
\end{aligned}
$$

So, the initial differential equation is turned to the algebraic one. The free model parameters are eliminated, so the evolution equation takes a universal form and may be compared with other models as well as tested to correspond to the observations data. In addition, the used kinematic characteristics are free from the uncertainties arising when physical quantities such as, for example, energy densities are measured.

\section{RESULTS}

We applied the described method to treat the two considered cosmological models. For the first proposed model (3) the evolution equation (2) reads

$$
2 \dot{H}+3 H^{2}-\sigma \mathrm{H}=0 .
$$

Using relation (7) we express the free parameter of the model $\sigma$ in a simple way through the Hubble parameter and the deceleration parameter:

$$
\sigma=H(1-2 q) .
$$

The second considered model (5) leads to the new parameterization of the vacuum dark energy [8] that together with the second Friedmann equation and the continuity condition results in time-dependence of the Hubble parameter $H \sim 1 / t$. It gives the following phenomenological quadratic parameterization of the cosmological constant density

$$
\Lambda(H)=\Lambda_{0}+3 \beta H^{2},
$$

where there are two free parameters $\Lambda_{0}$ and $\beta$. Hereby, the evolution equation (2) takes the form

$$
\dot{H}=\frac{\Lambda_{0}}{2}-\delta H^{2},
$$

where $\delta$ is a constant defined as $\delta=3 / 2(1-\beta)$. We repeat our procedure and differentiate the evolution equation using expressions (7) and (8) for the time derivatives of the Hubble parameter. Finally, we solve a simple system of algebraic equations and obtain free parameters of the proposed model in terms of the cosmographic ones

$$
\begin{gathered}
\delta=\frac{2+3 q+j}{2(1+q)} \text { or } \beta=\frac{1-j}{3(1+q)} \\
\Lambda_{0}=H^{2} \frac{j-q-2 q^{2}}{1+q} .
\end{gathered}
$$

Thus, having treated all the parameters of our models through the directly measurable cosmographical values, we can substitute the experimentally measured current values of the Hubble, the deceleration and the jerk parameters $H_{0}$, $q_{0}, j_{0}$ corresponding to the current time $t_{0}$. 
After that, the further solution of the evolution equations can be done with considering these dependences. The solution of the evolution equation (10) for the first model is given by the following time-dependence of the Hubble parameter

$$
\mathrm{H}(t)=\frac{H_{0} \sigma}{3 H_{0}+\left(\sigma-3 H_{0}\right) e^{-\frac{\sigma\left(t-t_{0}\right)}{2}}},
$$

where $H\left(t_{0}\right)=H_{0}$ is taken as an initial condition.

For the second model, it is also possible to solve the evolution equation (13) and to find the time-dependence of the Hubble parameter

$$
\mathrm{H}(t)=\sqrt{\frac{\Lambda_{0}}{2 \delta}} \operatorname{Tanh}\left[\sqrt{\frac{\delta \Lambda_{0}}{2}}\left(t-t_{0}\right)+\operatorname{ArcTanh}\left(\sqrt{\frac{2 \delta}{\Lambda_{0}}} H_{0}\right)\right]
$$

In (16), and (17), model parameters $\sigma, \delta$, and $\Lambda_{0}$ are correspondingly treated through $H_{0}, q_{0}$, and $j_{0}$ (11), (14-15).

The current values of $H_{0}, q_{0}$, and $j_{0}$ obtained from the fitting of observations data can be found, for example, in [16-18]. The recent analysis by Heneka [16] involves the comparison of the measurements for two cases: JLA compilation of SN Ia and the Pantheon sample. These measurements give different results for the deceleration parameter and the jerk: $q_{0}=-0.70 \pm 0.18$ and $j_{0}=0.52(+0.58-0.60)$ for the first case and respectively $q_{0}=$ $-0.86 \pm 0.07$ and $j_{0}=1.13 \pm 0.26$ for the second one. Having these parameters, we now can build the timedependences of the Hubble parameter and compare the results for two different models. For some typical range of values, the time-dependences of the Hubble parameter are depicted in Fig. 1.

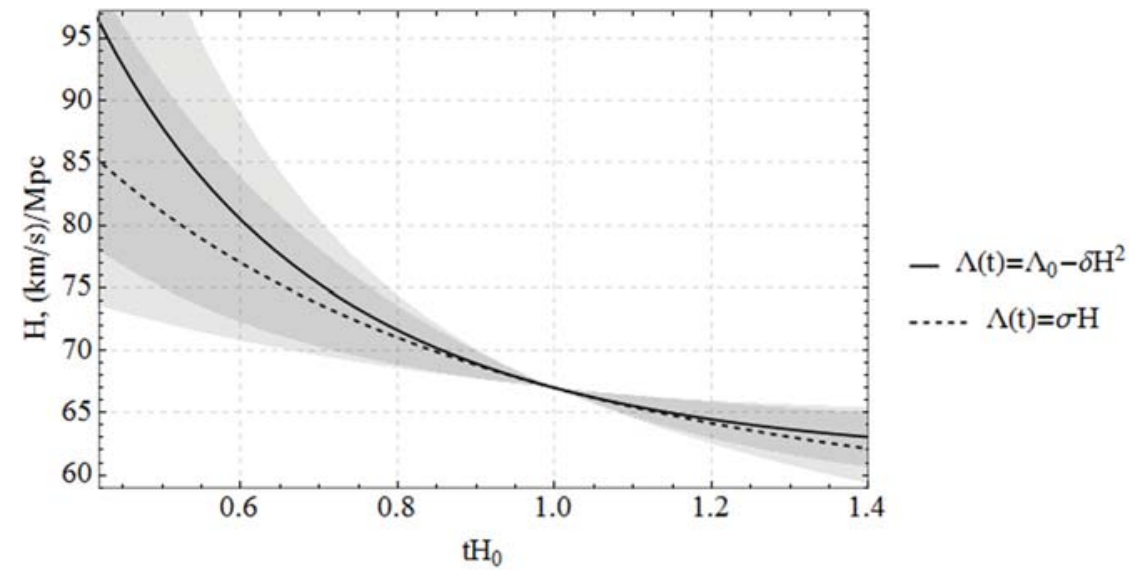

Fig. 1. Time-dependences (16) (dashed line) and (17) (solid line) of the Hubble parameter corresponding to two different models (3) and (12). A typical range of current values of the deceleration parameter is indicated by the gray area. Here, the current parameter value $q_{0}$, varies from -0.9 to -0.6 with the central value -0.75 . The Hubble constant $H_{0}$ is set to be $67\left(\frac{\mathrm{km}}{\mathrm{s}}\right) / \mathrm{Mpc}$, and the current value for jerk $j_{0}$ is set to be 1 .

As can be seen from the plot, the Hubble parameter decreases in time. The two considered models predict a similar behavior for this dependence. However, one can notice the difference for early times and late times, which is quite simple to explain. Indeed, for the case of $j_{0}=1$ we have $\beta=0$ (14) that means $\Lambda=\Lambda_{0}$ for the model (5). So, the model reduces to a constant term in the evolution equation (2), that affects the behavior of the derivative. While the other model (3) has there a linear positive addend $\sigma H$ that is greater for larger $H$ (and thus for the early times), so the derivative $\dot{H}$ is greater for early times and smaller for later times. In general, when $j_{0} \neq 1$, the character of the dependence for the model (12) may be different due to the appearing quadratic term.

\section{DISCUSSION}

Finally, it is possible not only to treat the model parameters, but also to rewrite Friedmann equations themselves in terms of directly observable cosmographic values. Dunajski and Gibbons [15] showed that for the three-parametric model in the case of the pressure-free matter and the cosmological constant, the evolution equation treated through cosmographic parameters yields 


$$
k=a^{2} H^{2}(j-1),
$$

where $\mathrm{k}$ (the spatial curvature) is regarded as a parameter. In particular if $k=0$, this relation reduces to a third order ODE

$$
j=1 .
$$

This constant jerk condition is consistent with almost all recent cosmological observations. Therefore, treating in this manner the Friedmann equations, we can obtain some simple restrictions on the cosmographic parameters. It allows testing the model for accordance with the current parameters relations received from observations.

We can perform the same procedure for the two considered models. For the first proposed model (3) the evolution equation (10) can be rewritten as

$$
2 \frac{\dot{H}}{H}+3 H-\sigma=0
$$

We differentiate it with respect to the cosmological time. Then, using expressions (7) and (8) for the time-derivatives of the Hubble parameter, we get by analogy to (19) a restriction on the jerk, involving already two cosmographic values:

$$
j=q^{2}+\frac{q+1}{2}
$$

This relation is equivalent to the Friedmann equation and enables us to test the correspondence of the proposed approach to the real values received from observations. The obtained constraint (21) compared with the ranges of current values from the mentioned observations are shown on the Fig.2. As can be seen, despite the simplicity and the absence of a strict physical background in this model, the result is in some accordance with the current cosmological observations.

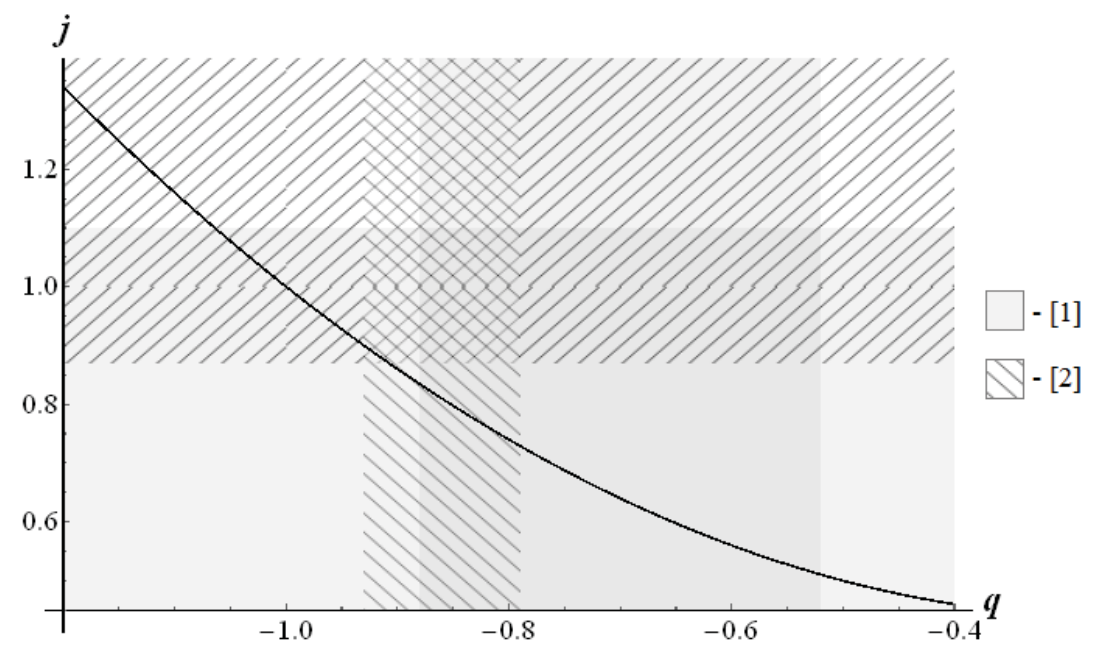

Fig. 2. Relation (21) for the jerk and the deceleration (black line) and areas of currently observable parameters values. Area [1] corresponds to the data from JLA compilation of SN Ia $\left\{q_{0}=-0.70 \pm 0.18\right.$, and $\left.j_{0}=0.52(+0.58-0.60)\right\}$. Area [2] is related to the Pantheon sample $\left\{q_{0}=-0.86 \pm 0.07\right.$ and $\left.j_{0}=1.13 \pm 0.26\right\}$. See [16] for details.

For the second proposed model (12) this procedure requires differentiating the evolution equation (13) two times to eliminate both free parameters. Using (7-9) for three time-derivatives of the Hubble parameter, we obtain the relation for the snap parameter $s$ that comes from the third derivative

$$
s=-\frac{j^{2}+j\left(q^{2}+4 q+1\right)+q(2 q+1)}{1+q}
$$

For the typical values $q_{0} \approx-0.75$, and $j_{0} \approx 1$ the estimation gives $s_{0} \approx 0.25$. A possible range of values of the snap parameter for different $q_{0}$, and $j_{0}$ are depicted in Fig. 3 . 


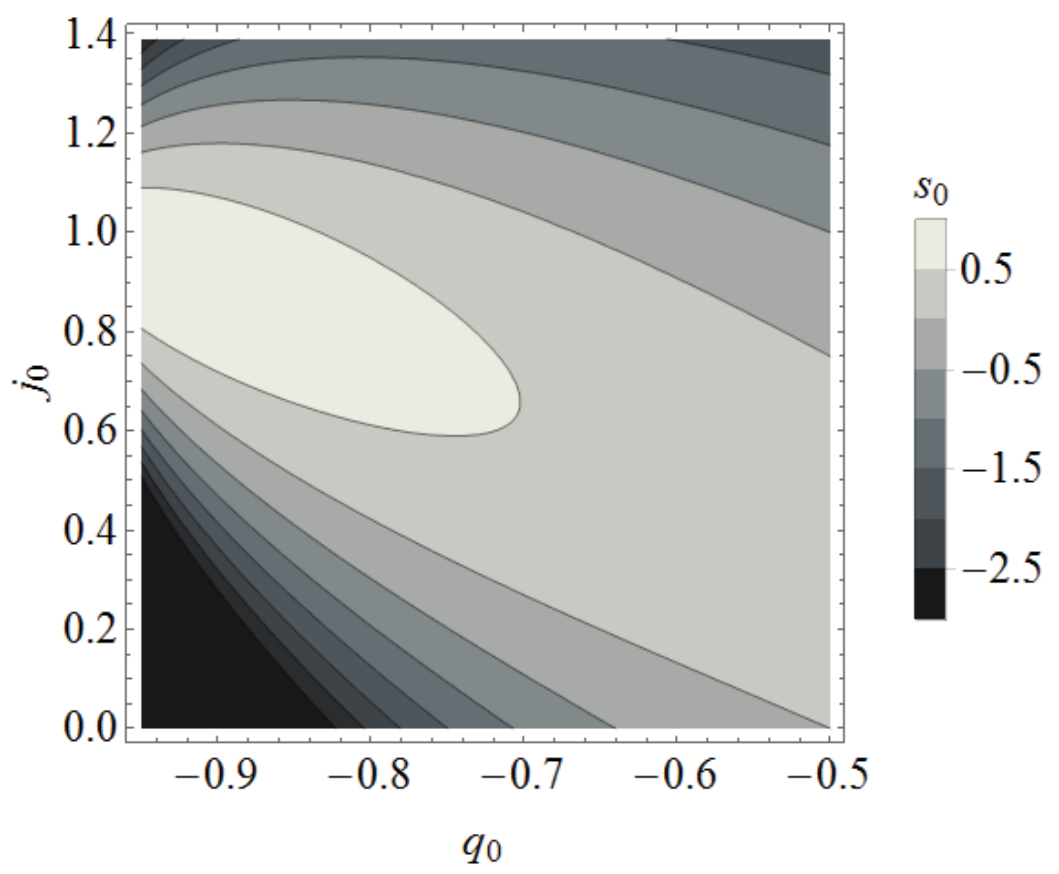

Fig. 3. The color diagram depicting the current value of the snap parameter (22) for some typical range of values for the deceleration parameter and the jerk.

\section{CONCLUSIONS}

Numerous cosmological models include multiple parameters of different nature that often are in complicated relations with the observed ones. It is possible to establish relations between parameters on a deeper level, thereby reducing their number to the unique parameter set. An important methodological principle, the so-called the Occam's razor, states: there is no need to multiply essences without necessity. This is more than an aesthetic question. Having reduced the number of cosmological parameters and developed a unified method of their determination, we will facilitate testing of the models and limit the role of the observational cosmology to the determination of a small number of the cosmographic parameters.

In the current paper, we have considered the algorithm of treating the free parameters of cosmological models in terms of the directly observable cosmographic values. We have shown that this method is especially efficient in the cases where the dynamical variables of the model directly depend on the Hubble parameter (the models with the timedependent cosmological constant in the form $\Lambda(t) \rightarrow \Lambda(H)$ ). Here, the cosmographic analysis in terms of the Hubble parameter derivatives $\dot{H}, \ddot{H}, \dddot{H} \ldots$ is more usable than the original approach of Dunajski and Gibbons [15].

The expressed model parameters can be then substituted in the solution of the evolution equation. Such a solution is easy to compare with other results and to test in the correspondence to the modern observation data.

Moreover, the method enables us to eliminate at all free parameters from the model and to obtain the Friedmann equation in form of some constraints on directly observable cosmological scalars. It allows considering the overlapping of the regions of cosmographic parameters for different models carrying different physical sense to analyze the reasonability of one or another approach. The obtained relations are exact and does not require any additional neglect. They lead us to work with simple algebraic equations instead of to solve the ODEs. The validity of resulting constraints can be verified by observations, thus providing the experimental test for any considered model.

\section{ACKNOWLEDGEMENTS}

I express my sincere gratitude to my scientific supervisor Yuri Bolotin for the valuable discussions and the help in the work.

Oryna Ivashtenko (D) https://orcid.org/0000-0002-2805-9405

\section{ORCID IDs}

\section{REFERENCES}

[1]. S. Dodelson, Modern cosmology, 4nd ed. (San Diego, CA: Academic Press, 2008). ISBN 978-012219141.

[2]. P.A.R. Ade et al., arXiv:1303.5076 [astro-ph.CO].

[3]. P.J.E. Peebles, Principles of Physical Cosmology (Princeton University Press, 1993).

[4]. H.E.S. Velten, R.F. vom Marttens and W. Zimdahl, Eur. Phys. J. C, 74, 3160 (2014)

[5]. Yu. L. Bolotin, D.A. Yerohin and O.A. Lemets, Physics-Uspekhi, 182(9), 941-986 (2012). (in Russian)

[6]. Yu. L. Bolotin, L. G. Zazunov, M. I. Konchatnyi, and O. A. Lemets, Odessa Astronomical Publications. 30, 13-15 (2017). 
[7]. S. Carneiro, M.A. Dantas, C. Pigozzo, and J.S. Alcaniz, Phys. Rev. D. 77, 083504 (2008).

[8]. M. Szydłowski, Phys. Rev. D. 91, 123538 (2015).

[9]. S. Coleman, Phys. Rev. D. 15(10), 2929-2936 (1977).

[10]. C.G. Callan, S. Coleman, Phys. Rev. D. 16(6), 1762-1768 (1977).

[11]. S. Coleman, F. De Luccia, Phys. Rev. D. 21(12), 3305 -3315 (1980) .

[12]. S. Weinberg, Gravitation and Cosmology (Wiley, Chichester, 1972). ISBN 0471925675.

[13]. A. Sandage, Astrophysical Journal. 136(2), 319-333 (1962).

[14]. M. Visser, arXiv:gr-qc/0411131.

[15]. M. Dunajski, G.W. Gibbons, DAMTP. 58 (2008).

[16]. C. Heneka, MNRAS. (2018).

[17]. A.G. Riess, A.V. Filippenko, P. Challis, A. Clocchiatti, A. Diercks, P.M. Garnavich, R.L. Gilliland, C.J. Hogan, S. Jha and R.P. Kirshner, The Astronomical Journal. 116(3), 1009 (1998).

[18]. A. Al Mamon and S. Das, Eur. Phys. J. C. 77, 495 (2017). 